\title{
Indices de equilibrio $y$ desequilibrio para el análisis y diagnóstico territorial
}

\section{Indexes of balance and imbalance to the analysis and territorial diagnosis}

Prof. Dr. Manuel Doval Abad.

Contacto: manuel.doval@upm.es

Universidad Politécnica de Madrid

ETSAM/UPM

Avda. Juan de Herrera 4 Madrid 28040

\begin{abstract}
The indexes detailed in this article are designed to assess morphological, demographical and economically the system of urban cores of a territory, in its local, provincial and regional scale, as well as their interactions, from the point of view of their marginality, centrality and mutual influences. These indexes can be applicable to the analysis, planning and management of any territory, particularly on those cases where the development inequalities are highly pronounced.
\end{abstract}

Keywords: indexes, centrality, territory, inequalities.

\section{Introdución}

Los diferentes grados de concentración de factores de producción, nivel de recursos, tecnología, renta, población, oportunidades de empleo y acceso a servicios y equipamientos, presentes en gran parte de los países desarrollados y en desarrollo, derivados de La lógica acumulativa y competitiva de los mercados en el modo de producción y distribución vigente (generalizado a escala planetaria), reflejan y potencian la existencia de sociedades duales, de riqueza y pobreza, en función de su ubicación geográfica, efectos frontera positivos o negativos, inercias históricas de dominación o precariedad, perpetuándose y ampliándose en el neoliberalismo contemporáneo, los

\section{Resumen}

Los índices que se detallan en este artículo están diseñadas para evaluar morfológica, demográfica y económicamente el sistema de núcleos urbanos de un territorio, en su escala local, provincial y regional, así como sus interacciones, desde el punto de vista de su marginalidad, centralidad e influencias mutuas. Estos índices pueden ser aplicables al análisis, planeamiento y gestión de cualquier territorio, en particular en aquellos casos en que las desigualdades de desarrollo son muy pronunciadas

Palabras clave: índices, centralidad, territorio, desigualdades desequilíbrios $\quad$ y disparidades territoriales.

Estas disparidades se pueden observar en todos los ámbitos, desde el internacional, con la constitución de metrópolis globales, centralizadoras de poder político, económico, tecnología y conocimiento, hasta el nacional, regional, o local, con la asignación y asunción de roles en la división territorial de la producción y el consumo.

Bajo esas premisas, la intervención de los poderes públicos se hace precisa para disminuir las diferencias espaciales, paliar marginalidades y buscar un desarrollo regional y local equilibrado y sostenible. 
El presente artículo trata sobre los índices que valoran las características y relaciones entre diferentes núcleos urbanos regionales con influencia metropolitana que permitan diagnosticar los equilibrios y desequilibrios territoriales existentes.

\section{Indices De Concentración Económica y Poblacional}

\section{Índice de Primacía-Regla Rango-Tamaño}

Para analizar el nivel de equilibrio en el sistema de ciudades de un área referido a la distribución de los tamaños, con influencia en la difusión del desarrollo económico, utilizamos los Índices de

Primacía. $^{\text {i que se obtienen }}$ mediante el cociente entre la población de la ciudad mas poblada y la suma de esta población y las poblaciones de las tres siguientes del área considerada.

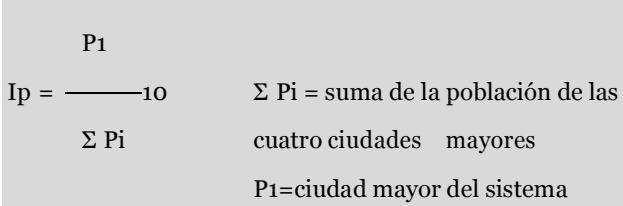

Los valores de este índice oscilan porcentualmente entre 25 y 100.

Cuando son sistemas macrocéfalos, en el que una gran parte de la población y la actividad económica de la región se halla más concentrada, el valor del índice de primacía es alto. Mientras en el caso de los sistemas bicéfalos o tricéfalos se obtienen valores bajos.

En el caso del conjunto de España el Índice de primacía es del 50,9\%, debido a que la diferencia de tamaño entre Madrid como metrópoli principal y la tercera y cuarta ciudad es cuatro veces mayor, pudiendo considerarse asimilable a un sistema bicéfalo al encontrase Barcelona en un punto intermedio.

La Comunidad de Madrid, tiene un índice de primacía muy elevado, Ip = 84,2 con un sistema con tendencia a ser macrocéfalo, donde su capital abarca 16 veces más población que cualquiera de las tres ciudades restantes del sistema.

Relacionando el Índice de Concentración de la población de la capital respecto a la provincia (Icp) con el Índice de Primacía (Ip) se obtiene el valor de un nuevo índice IED que refleja el grado de equilibriodesequilibrio en los asentamientos.



Gráfico 1 Indices de Primacía en España

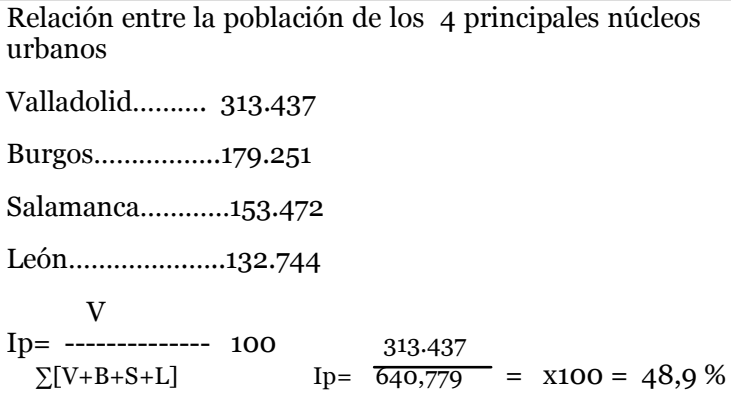

Cuadro 1: Índice de Primacía de Castilla y León - elaboración propia (INE 2011)

Aplicando este análisis estático coyuntural (referido a datos actuales), a un análisis dinámico evolutivo, se obtendría una radiografía estructural de las tendencias históricas del sistema de asentamientos en una región ; y dada la intrínseca relación

entre población y economía, aplicando este mismo método de análisis a la distribución de los factores de producción en el territorio, encontraríamos valores similares en el aspecto económico regional.

Conceptualmente cercano a este índice, está la evaluación de las relaciones entre ciudades de un área urbana mediante la regla RangoTamaño. Según esta regla se puede determinar el peso demográfico de cualquier núcleo urbano en un sistema 
dado, conociendo el rango que ocupa dentro de dicho sistema y la población de la mayor ciudad del área considerada, mediante la fórmula:

$\mathrm{Pr}=\mathrm{P} 1 / \mathrm{r}$

$\mathrm{P}_{\mathrm{r}}=$ población de la ciudad de rango $\mathrm{r}$.

$\mathrm{P}_{1}=$ población de la mayor ciudad.

$\mathrm{r}=$ rango de la ciudad cuya población pretendemos conocer.

Si expresamos gráficamente los cocientes entre la población obtenida y la esperada de un determinado sistema de ciudades, podemos apreciar que, en general, las distribuciones más equilibradas se dan en los países con mayor desarrollo, debido a la existencia en ellos de mallas urbanas jerarquizadas y complementarias.

Valores del cociente próximos a la unidad indicarían un alto nivel de equilibrio territorial, valores por encima señalarían una población real superior a la esperada, y valores inferiores a uno describirían ciudades con población por debajo de las que cabría esperar en una situación ideal. Aplicado a las cuatro ciudades mayores de Castilla y León, podemos ver en los cuadros 1 y 2 los desequilibrios existentes, en particular referidos a la tercera y cuarta ciudad de la Región.

\begin{tabular}{|l|l|l|l|}
\hline & $\begin{array}{l}\text { Población } \\
\text { real } \\
\text { observada } \\
\text { (Po) hab. }\end{array}$ & $\begin{array}{l}\text { Población } \\
\text { esperada } \\
(\mathrm{Pe})\end{array}$ & Cociente \\
& & $\mathrm{Po} / \mathrm{Pe}$ \\
\hline hab. & \\
\hline 1.-Valladolid & 313.437 & $\begin{array}{l}313.437 / 1= \\
\mathbf{3 1 3 . 4 3 7}\end{array}$ & $\mathbf{1 , 0 0}$ \\
\hline 2.-Burgos & 179.251 & $\begin{array}{l}313.437 / 2= \\
\mathbf{1 5 6 . 7 1 9}\end{array}$ & $\mathbf{1 , 1 4}$ \\
\hline Salamanca & 153.472 & $\begin{array}{l}313.437 / 3= \\
\mathbf{1 0 4 . 4 7 9}\end{array}$ & $\mathbf{1 , 4 7}$ \\
\hline 4.-León & 132.744 & $\begin{array}{l}313.437 / 4= \\
\mathbf{1 7 8 . 3 5 9}\end{array}$ & $\mathbf{0 , 7 4}$ \\
\hline
\end{tabular}

Cuadro2: Castilla y León (España) ii

\begin{tabular}{l|l|l|l} 
Ciudad & $\begin{array}{l}\text { Población } \\
\text { real } \\
\text { observada } \\
\text { (Po) hab. }\end{array}$ & $\begin{array}{l}\text { Población } \\
\text { esperada }\end{array}$ & Cociente \\
& $(\mathrm{Pe})$ & Po/Pe \\
hab. & \\
\hline 1.-Madrid & 3.265 .038 & 3.265 .038 & $\mathbf{1 , 0 0}$ \\
\hline 2.-Barcelona & 1.615 .448 & $\mathbf{1 6 3 2 5 1 9}$ & $\mathbf{0 , 9 9}$ \\
\hline 3.-Valencia & 798.033 & $\mathbf{1 . 0 8 8 . 3 4 6}$ & $\mathbf{0 , 7 3}$ \\
\hline 4.-Sevilla & 703.021 & $\mathbf{8 1 6 . 2 5 9}$ & $\mathbf{0 , 8 6}$
\end{tabular}

Cuadro 3: España ( INE 2011)

La situación en el conjunto de España señalada en el cuadro 3 expresa un sistema bicéfalo (Madrid, Barcelona) con cierto desequilibrio referido a la tercera y cuarta ciudad en el rango de ciudades del Estado.

Referido a Brasil, Zárate Martín y Rubio Benito iii muestran en las ocho principales ciudades del Estado la situación demográfica que se refleja en el Cuadro 4 y en el Gráfico 2, donde se aprecian que los mayores desequilibrios se producen respecto a las ciudades de Salvador y Belo Horizonte, con una cierta simetría con el caso español considerando la bicefalia Sao Paulo/Río.

\begin{tabular}{|l|c|c|c|c|}
\hline \multicolumn{1}{|c|}{ Ciudad } & Rango & $\begin{array}{c}\text { Población } \\
\text { real } u \\
\text { observada } \\
\left(\mathrm{P}_{\mathrm{o}}\right)\end{array}$ & $\begin{array}{c}\text { Población } \\
\text { esperada } \\
\left(\mathrm{P}_{\mathrm{e}}\right)\end{array}$ & $\mathrm{P}_{\mathrm{o}} / \mathrm{P}_{\mathrm{e}}$ \\
\hline $\begin{array}{l}\text { Sao } \\
\text { Paulo }\end{array}$ & 1 & 10.434 .252 & 10.434 .252 & $1^{\prime} 00$ \\
\hline $\begin{array}{l}\text { Río de } \\
\text { Janeiro }\end{array}$ & 2 & 5.857 .904 & 5.217 .126 & $1^{\prime} 12$ \\
\hline Salvador & 3 & 2.443 .107 & 3.478 .084 & $0^{\prime} 70$ \\
\hline $\begin{array}{l}\text { Belo } \\
\text { Horizonte }\end{array}$ & 4 & 2.238 .526 & 2.608 .563 & $0^{\prime} 86$ \\
\hline Fortaleza & 5 & 2.141 .402 & 2.086 .850 & $1^{\prime} 03$ \\
\hline Brasilia & 6 & 2.051 .146 & 1.739 .042 & $1^{\prime} 18$ \\
\hline Curitiba & 7 & 1.587 .315 & 1.490 .607 & $1^{\prime} 06$ \\
\hline Recife & 8 & 1.422 .905 & 1.374 .684 & $1^{\prime} 04$ \\
\hline
\end{tabular}

Cuadro 4: Rango -Tamaño en las ciudades brasileñas (2001)

Fuente: Zárate Martín y Rubio Benito 2005

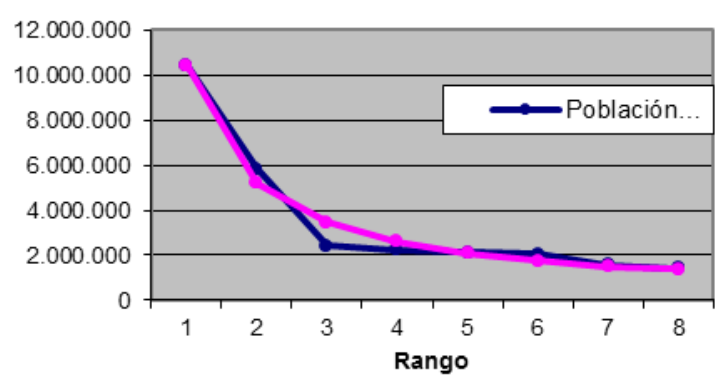

Gráfico 2: Sistema de ciudades en Brasil

Fuente: Zárate Martín y Rubio Benito 2005 
Indice de Disimilaridad ${ }^{\mathrm{iv}}$

\section{DISTRIBUCIÓN TERRITORIAL DE LA POBLACIÓN}

Se obtiene el Índice de Disimilaridad mediante la fórmula:

$$
\text { Id = \% Sup. - \% Pob. }
$$

Los valores resultantes de esa ecuación miden la distribución de una determinada población en un territorio concreto. Un índice Id aproximándose a o implicaría una población uniformemente repartida en el espacio, y un valor alejado de cero reflejaría un mayor desequilibrio. La evolución de estos índices a lo largo del tiempo expresaría las tendencias en cuanto a la distribución espacial de la población. Si los valores son $>0$, se trata de una ciudad con un mayor porcentaje de superficie que de población y por tanto estamos ante una población dispersa. Si los valores son $<0$, se trata de ciudades con un alto grado de concentración de población respecto a la superficie que poseen.

\section{EVOLUCIÓN DEL ÍNDICE DE DISIMILARIDAD EN CAPITALES DE PROVINCIA}

\begin{tabular}{l|ccccc|c|c|}
\hline \multicolumn{1}{|c}{ CAPITAL } & \%SUP. & $\%$ \%ОB.01 & $\%$ \%OB.09 & ID. 2001 & ID. 2009 & POBLACIÓN & TENDENCIA \\
\hline VALLADOLID & 15,3 & 30,0 & 29,2 & $-14,8$ & $-13,9$ & CONCENTRADA & UNIFORMIDAD \\
\hline SALAMANCA & 3,0 & 14,8 & 14,3 & $-11,8$ & $-11,3$ & CONCENTRADA & UNIFORMIDAD \\
\hline LEÓN & 3,0 & 12,4 & 12,3 & $-9,4$ & $-9,3$ & CONCENTRADA & SIN CAMBIOS \\
\hline BURGOS & 8,3 & 15,8 & 16,4 & $-7,5$ & $-8,2$ & CONCENTRADA & CONCENTRACIÓN \\
\hline PALENCIA & 7,3 & 7,6 & 7,6 & $-0,3$ & $-0,3$ & UNIFORME & SIN CAMBIOS \\
\hline ZAMORA & 11,5 & 6,2 & 6,1 & 5,4 & 5,4 & DISPERSA & SIN CAMBIOS \\
\hline SEGOVIA & 12,6 & 5,2 & 5,2 & 7,5 & 7,4 & DISPERSA & SIN CAMBIOS \\
\hline ÁVILA & 17,9 & 4,7 & 5,2 & 13,2 & 12,7 & DISPERSA & UNIFORMIDAD \\
\hline SORIA & 21,0 & 3,3 & 3,6 & 17,7 & 17,4 & DISPERSA & UNIFORMIDAD
\end{tabular}

Cuadro 5: Indice de Disimilaridad en Castilla y León

El cuadro 5 refleja en el caso de la región española de Castilla y León, el nivel de concentración o dispersión poblacional de las capitales de provincia, así como las tendencias observadas en el periodo 2001-2009.

Este índice es muy similar al índice de segregación ${ }^{v}$, que mide la distribución de un grupo de población en el espacio urbano, tendiendo a valor cero las distribuciones igualitarias y a valor 1 la segregación máximavi.

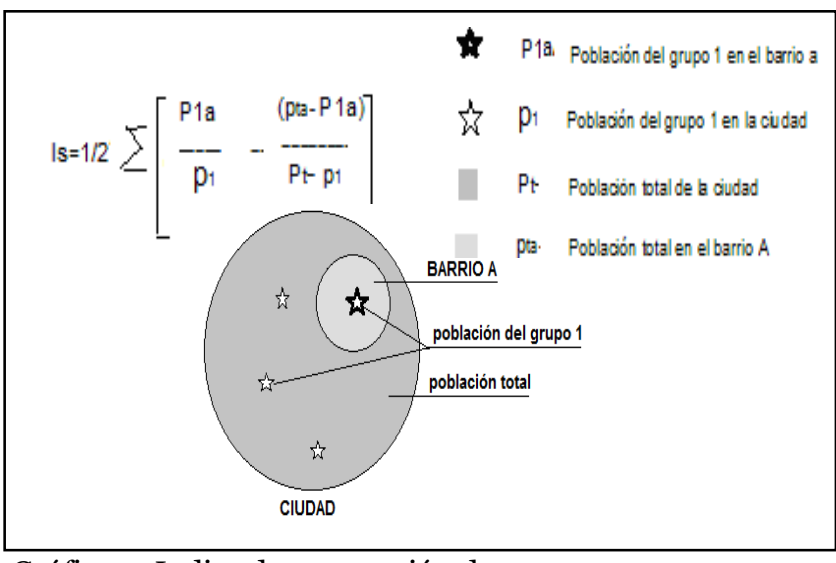

Gráfico 3: Indice de segregación de un grupo social en la ciudad 


\section{Índice de Gini vii}

\section{DISTRIBUCIÓN REAL Y UNIFORME}

El índice de Gini se utiliza para medir cualquier forma de distribución desigual. Desde el punto de vista urbanístico lo usamos para calcular niveles de concentración de actividad y población en determinados puntos del espacio.

Este índice es similar al índice de disimilaridad pero un poco más complejo, ya que mide la relación entre la distribución real (de la población en este caso) y una situación hipotética de uniformidad total entre las ciudades comparadas. Sus valores oscilan entre cero y uno.$$
\mathrm{IG}=(\mathrm{p}-\mathrm{s}) / \mathrm{p}
$$$$
\mathrm{p}=\text { porcentaje acumulado de población }
$$$$
\mathrm{s}=\text { porcentaje acumulado de superficie }
$$

-Si el índice de Gini, IG= o , sería el caso ideal en el que todos los municipios están equilibrados y su población aumenta o disminuye proporcionalmente a su superficie. Calculando el índice de Gini en el caso real, IG, y en el caso hipotético de desequilibrio total, IGD, en el que toda la población estuviese concentrada en un municipio:
}

\begin{tabular}{|c|c|c|c|c|c|c|c|c|}
\hline \multirow{2}{*}{ CAPITAL } & \multirow{2}{*}{ POB. } & \multicolumn{3}{|c|}{ POB. ACUMULADA } & \multirow{2}{*}{$\begin{array}{l}\text { SUP. } \\
\left(\mathrm{km}^{2}\right)\end{array}$} & \multicolumn{3}{|c|}{ SUP. ACUMULADA } \\
\hline & & Hab. & $\%$ & pi. & & $\left(\mathrm{km}^{2}\right)$ & $\%$ & si. \\
\hline Valladolid & 317.864 & 317.864 & 29,2 & $\mathbf{2 9 , 2}$ & 197,9 & 197,9 & 15,3 & 15,3 \\
\hline Burgos & 178.966 & 496.830 & 16,4 & 45,6 & 107,1 & 305,0 & 8,3 & 23,6 \\
\hline Salamanca & 155.619 & 652.449 & 14,3 & 59,9 & 39,3 & 344,3 & 3,0 & 26,6 \\
\hline León & $134 \cdot 305$ & 786.754 & 12,3 & 72,3 & 39,0 & 383,4 & 3,0 & 29,6 \\
\hline Palencia & 82.651 & 869.405 & 7,6 & 79,8 & 94,7 & 478,1 & 7,3 & 36,9 \\
\hline Zamora & 66.293 & 935.698 & 6,1 & 85,9 & 149,3 & 627,3 & 11,5 & 48,5 \\
\hline Ávila & 56.855 & $\begin{array}{l}992.553 \\
1.049 .2\end{array}$ & 5,2 & 91,2 & 231,9 & 859,2 & 17,9 & 66,4 \\
\hline Segovia & 56.660 & 13 & 5,2 & 96,4 & 163,6 & $1.022,8$ & 12,6 & 79,0 \\
\hline Soria & 39.528 & 1.088 .741 & 3,6 & 100,0 & 271,8 & $1.294,6$ & 21,0 & 100,0 \\
\hline TOTAL & & $p=\Sigma p i=66$ & 35 & & & $\mathbf{s}=\Sigma \mathbf{s i}$ & $=425,81$ & \\
\hline
\end{tabular}

Cuadro 6; Poblaciones y superficies acumuladas en las ciudades de Castilla y León

Fuente: Elaboración propia. INE 2009

IG $=660,35-425,81 / 660,35=\mathbf{0 , 3 5 5}$ IGD $=900-425,81 / 900=\mathbf{0 , 5 2 7}$

Este resultado indica que el conjunto de estas ciudades tiene una distribución de la población en su superficie no equilibrada entre ellas, no estando relacionada la población proporcionalmente a la superficie de cada una.

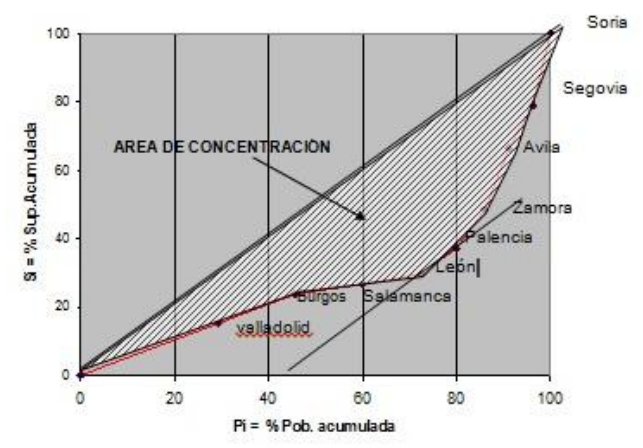

Gráfico 4: Curva de Lorentz 
La representación gráfica del Indice de Gini es la Curva de Lorentz, ejemplificada en el gráfico 4 para el caso de Castilla y León en España.

- El área rallada en la gráfica es el "área de concentración”. Cuanto menor es su superficie, acercándose a la diagonal de la tabla de $45^{\circ}$, menor es el índice de Gini y por tanto hay más equilibrio entre población-superficie en las ciudades.

- Dos ciudades mantendrán la misma proporción en el incremento de ambos factores (población y superficie) si la pendiente que crean es paralela a la diagonal. Si el ángulo es inferior a $45^{\circ}$ es debido a un mayor incremento de la población, mientras que si el ángulo es superior a $45^{\circ}$ el incremento de la superficie es mayor al de la población. Datos coincidentes con las conclusiones obtenidas con el Índice de Disimilaridad.

En definitiva se puede cuantificar qué ciudades desequilibran más el conjunto observando el ángulo señalado en la gráfica o realizando el cálculo:

\% INCREMENTO DE POB. $=$ [ ( INCREM. \% POB / INCREM. \% SUP.) - 1] x 100

RESPECTO A LA SUP.

- los valores que tienden a cero son ciudades con mayor equilibrio entre su población y su superficie.

- los valores positivos son las ciudades donde el porcentaje de población supera al porcentaje de superficie.

A nivel socioeconómico, el Índice puede representar el nivel de desigualdad de rentas, donde el o es la situación más igualitaria y el 1 la de mayor desigualdad.
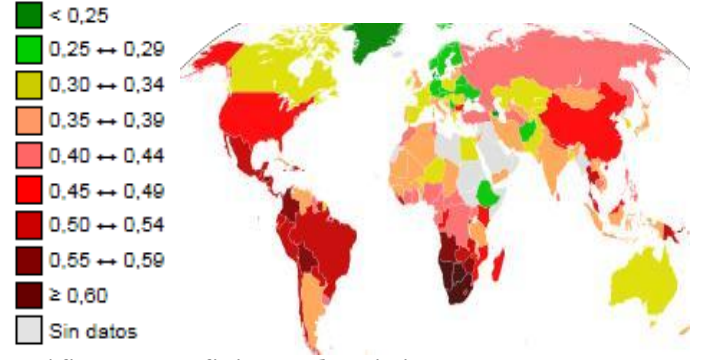

Gráfico 5: Coeficiente de Gini

Países según nivel de igualdad de ingresos

Fuente: Informe Naciones Unidas 2005

\begin{tabular}{cccc}
\multicolumn{4}{c}{$0=$ equilibrio total . 1 =desequilibrio total } \\
Castilla y León & ESPAÑA & BRASIL & Mundo \\
0,35 & 0,35 & 0,52 & 0,65
\end{tabular}

Cuadro 7: Indice de Gini referido al nivel de rentas

\section{Índice de Desequilibrio Poblacional}

Este índice está formado por el cociente entre el índice de Gini y el índice en desequilibrio total

(donde toda la población estuviese concentrada en un solo núcleo).

Este índice es el de aplicación más directa y muestra de forma clara el desequilibrio económico entre dos o más territorios 0 regiones, expresándose mediante el cociente de la variable considerada en las áreas comparadas (producto interior bruto, renta per cápita, etc.). A través del Indice de Gini podemos observar gráficamente la desigualdad de rentas existente.

Los desequilibrios de rentas se ofrecen dentro de cada país o región y tanto en países en desarrollo como en países desarrollados, si bien las disparidades en términos porcentuales son mayores dentro de determinadas áreas coincidentes con bajos índices de desarrollo humano.

En el caso de España como se observa en los gráficos 6 y 7 el desequilibrio de rentas en el conjunto del territorio nacional es significativo, con 7 regiones que superan el índice 100 de la renta media europea 
superan el umbral de empleo correspondiente en una determinada rama de actividad son calificadas como especializadas en esa actividad o diversificadas si no están especializadas en esa actividad.

El umbral se calcula añadiendo al valor del empleo normal (x medio) una desviación típica calculada mediante:



\begin{tabular}{|ll|}
\hline CIUDADES & Ramas de especialización \\
\hline $\begin{array}{l}\text { Segovia } \\
\text { Zamora }\end{array}$ & $\begin{array}{l}\text { Rama I (Agricultura) } \\
\text { Rama I (Agricultura) y } \\
\text { RamaIII(Construcción) }\end{array}$ \\
Burgos & Rama II (Industria) \\
León & Rama IV (Servicios) \\
Palencia & Diversificada \\
Salamanca & Rama IV (Servicios) \\
Soria & Rama I (Agricultura) \\
Valladolid & Rama II (Industria) \\
\hline
\end{tabular}

Cuadro 8: Especialización funcional de las ciudades de Castilla y León

Fuente: Elab. Propia a partir de los resultados obtenidos con el Método Nelson

\section{Indice de Christaller}

\section{CENTRALIDAD O INFLUENCIA DE LAS CAPITALES}

Un concepto práctico derivado de la jerarquía del lugar central es el de centralidad, entendiéndola como el excedente de infraestructura, servicios, y su aprovechamiento en beneficio de la población que se encuentra bajo el área de influencia de una localidad, la cual determina su jerarquía y operatividad ante el conjunto de localidades que se nterrelacionan con la misma. El índice está basado en la teoría de los lugares centrales de Christaller Walter (1933). Este factor se puede medir mediante la fórmula:

$$
\mathrm{C}=\mathrm{Ec}-(\mathrm{Hc} / \mathrm{Hr}){ }^{*} \mathrm{Er}=\mathrm{Ec}-\mathrm{Kr} * \mathrm{Er}
$$

$\begin{array}{cc}\text { Ec }=\text { Empleados en la ciudad } & \mathrm{Er}=\text { Empleados en la región } \\ \mathrm{Hc}=\text { Población de la ciudad } & \mathrm{Hr}=\text { Población de la región }\end{array}$ $\mathrm{Kr}=\mathrm{Hc} / \mathrm{Hr}$

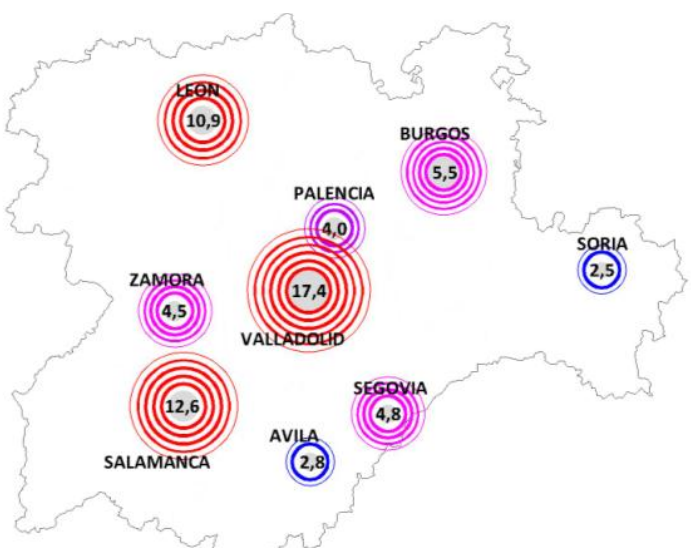

Gráfico 9: Indice de Christaller. Centralidad regional en Castilla y León

Cuanto mayor es el valor de $\mathrm{C}$, más alto será el grado de centralidad del lugar considerado.

\section{Indice de Davies (1967) ${ }^{x i i}$}

\section{CENTRALIDAD FUNCIONAL}

El valor resultante indica la centralidad de un núcleo de población en relación a la actividad analizada, sirve para cuantificar el número de empleos en una determinada rama de actividad y su concentración por comparación con los demás núcleos analizados. El cálculo del índice de centralidad se obtiene:

Empleos por rama de actividad en una ciudad

IC =Empleos por rama de actividad en el conjunto

Valores entre o y 1

Mayor valor $>$ Centralidad Funcional 


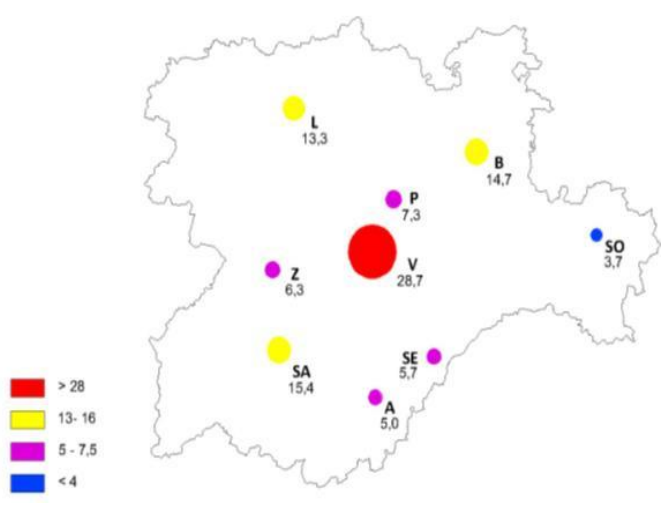

Grafico 10: Indice de centralidad según el empleo en Castilla y león. Sector servicios

\section{Indice de localización y empleo básico}

\section{ACTIVIDADES BÁSICAS Y NO BÁSICAS}

Este índice mide el valor por rama de actividad que tiene un núcleo respecto al conjunto, caracterizándose cada actividad en básica (orientada a la exportación) o no básica (orientada hacia el consumo interior $)^{\mathrm{xiii}}$.

Cociente de localización $(Q)$ para cada actividad en la ciudad considerada

$\mathrm{Q}=\frac{\text { ei } / \mathrm{e}}{\mathrm{Ei} / \mathrm{E}}=\frac{\text { Empleo por ciudad }}{\% \text { Empleo en el conjunto }}$
$\begin{aligned} & \text { ei = empleo en la actividad i en la ciudad } \mathrm{e}=\text { empleo total en la } \\ & \text { ciudad } \\ & \text { Ei = empleo en la actividad en la región } \\ & \mathrm{E}=\text { empleo total en la región }\end{aligned}$

En la aplicación práctica de este método, se pueden producir las siguientes situaciones:

a) Cuando Q es igual a 1, la ciudad se halla equilibrada en esa actividad, ya que la proporción de empleo en la ciudad y en el conjunto de ciudades es la misma.

b) Cuando Q es mayor de 1, la ciudad está especializada en esa actividad. La ciudad es exportadora en esa actividad, ya que produce más de lo que consume. c) Cuando Q es menor de la unidad la ciudad produce menos de lo que consume en la actividad considerada, por lo que se verá obligada a realizar importaciones.

Para el cálculo del empleo básico, Eb, por ciudad se utilizan los datos anteriores, pero en lugar del cociente se calcula la diferencia entre porcentajes de empleo por sector:

\% Eb rama-ciudad=e i / e - E i / E = \% Empleo por ciudad Empleo total

$\mathrm{N}^{\circ} \mathrm{Eb}=\%$ empleo básico $\mathrm{x} \quad \mathrm{n}^{0}$ total de empleos

Los valores (en $\mathrm{n}^{0}$ de empleos básicos) son negativos en las ramas de actividad en donde serían necesarios más empleos, para equilibrarse con el valor medio del conjunto de ciudades

estudiadas.

\section{Indice de Regularidad Perimetral}

\section{IDONEIDAD ENVOLVENTE}

Con este índice se mide la regularidad del perímetro de una forma territorial. Cuanto más irregular sea mayor diferencia de distancias del perímetro al centro geométrico $y$ por tanto, mayores dificultades en las comunicaciones, la distribución de equipamientos, expresando y propiciando la dispersión.

Se calcula dividiendo el diámetro del círculo circunscrito de la forma (Dc) entre el diámetro del mayor círculo inscrito (Di), obteniéndose resultados mayores a 1; siendo la forma idónea con valor 1, donde las dos áreas se igualarían: 


$$
\operatorname{Ir}=\mathrm{Dc} / \mathrm{Di}
$$

En el cuadro 9 se expresa el índice de regularidad de tres provincias de la región castellano-leonesa, observándose la coincidencia del mayor valor con el menor desarrollo.

\begin{tabular}{|l|c|c|c|}
\hline & $\begin{array}{c}\text { DIAMETRO } \\
\text { C. } \\
\text { CIRCUNSCRI } \\
\text { TO }(\text { Km) }\end{array}$ & $\begin{array}{c}\text { R. INSCRITO } \\
\text { (Km) }\end{array}$ & $\begin{array}{c}\text { RELACION } \\
\text { CIRCUNSCRITO/ } \\
\text { INSCRITO } \\
\text { (I regularidad) }\end{array}$ \\
\hline ÁVILA & 140 & 72 & 1,9 \\
SEGOVIA & 127 & 63 & 2,0 \\
ZAMORA & 178 & 74 & 2,4 \\
CASTILLA & & & 2,0 \\
Y LEÓN & 472 & 239 & 2,0
\end{tabular}

Cuadro 9: Índice de Regularidad CASTILLA Y LEÓN

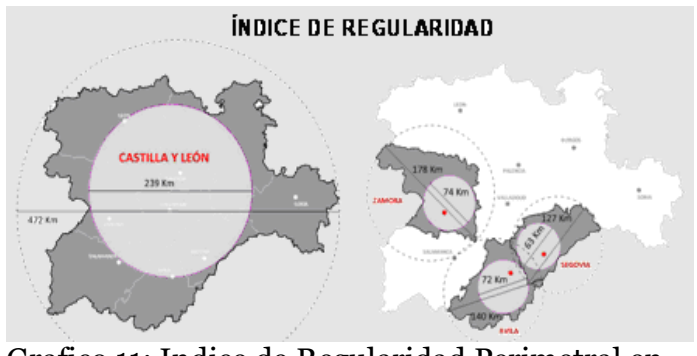

Grafico 11: Indice de Regularidad Perimetral en

Castilla y León

\section{Índice Superficial de forma}

\section{DISPERSIÓN TERRITORIAL}

Estudia las características morfológicas de un núcleo y sirve para analizar la compacidad de los crecimientos urbanos (teniendo en cuenta que muchos de ellos se ven condicionados por la topografía y otras barreras físicas), considerando que a mayor dispersión de la población mayores costes y peor accesibilidad a los servicios.

Este índice, calculados a través del cociente entre el área urbana real y la del círculo circunscrito, permiten conocer cuantitativamente la tipologia del crecimiento (concentración dispersión), com valore entre o y 1 , correspondiendo lós extremos a territórios lineales o circulares $\mathrm{y}$ considerando de mayor eficiência quellos los valores más cercanos a la unidad. Es también conocido como Índice de Cole, definiéndose por el cociente entre la superficie del núcleo $(\mathrm{Sn})$ y el área del círculo circunscrito (Sc):

$$
\mathrm{Is}=\mathrm{Sn} / \mathrm{Sc}
$$

INDICE DE FORMA = $1:$ núcleo más concentrado. INDICE DE FORMA = 0 : núcleo más disperso.

Cuanto menor es el valor del índice superficial de forma, menos compacto es el territorio y más disfuncionales son las distintas áreas y peor la accesibilidad a los distintos equipamientos y servicios. En el caso de Segovía, (gráfico 11) el suelo urbano calificado presenta un índice superficial de forma muy bajo, reflejando excesiva dispersión de la población.

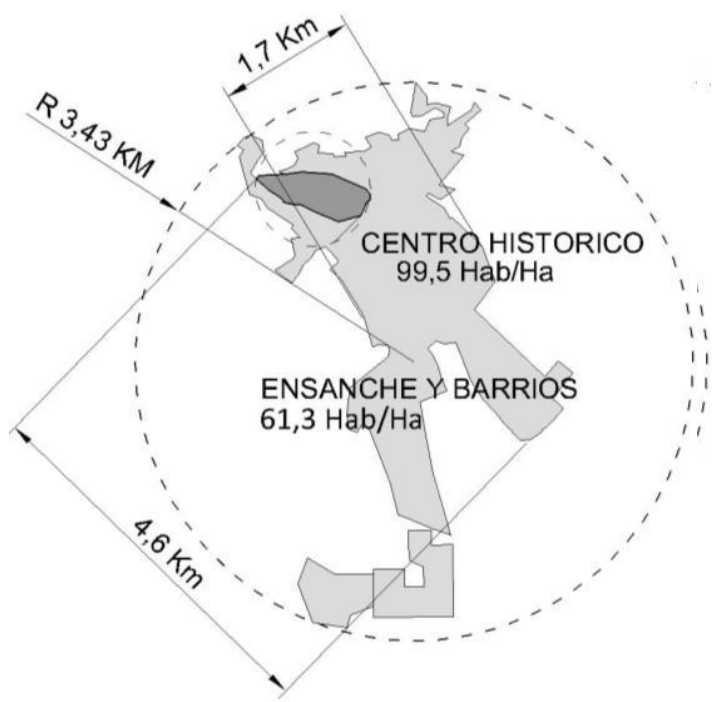

Gráfico 12:Suelo urbano - La distancia entre dos puntos extremos de la ciudad es de $6,9 \mathrm{~km}$ y una anchura media de $1,7 \mathrm{~km}$, por tanto posee una ELACIÓN LONGITUD - ANCHURA $=4,1$

INDICE DE FORMA - CIUDAD
DE SEGOVIA en CASTILLA Y LEON
Área del suelo urbano
Is $=\frac{=910 \text { has } / 3708 \text { Has }=0,25}{\text { Área del círculo circunscrito }}$

\section{Indice de Concentración - Dispersión .Rn ${ }^{x i i}$}

\section{DISTRIBUCIÓN ESPACIAL DE LOS ASENTAMIENTOS}

La medición de la distribución de los asentamientos en el espacio se realiza mediante el índice $\mathrm{Rn}$ : 
$\mathrm{Rn}=2 \times$ dmedio $\sqrt{ } \mathrm{N} / \mathrm{S}$

dmedio $=\Sigma \mathrm{d} / \mathrm{N}$

$\mathrm{d}=$ distancia media en línea recta de los núcleos respecto al más próximo entre ellos

$\mathrm{S}=$ superficie del territorio

$\mathrm{N}$ = número de asentamientos estudiados

El valor del índice $\mathrm{Rn}$ puede variar entre o y 2,15. En el primer caso se trataría de una distribución totalmente concentrada, en la que todos los asentamientos aparecerían en contigüidad. En el caso del índice mayor sería una distribución equilibrada. Y el caso más común con un índice con valores próximos a 1 se referiría a una dispersión aleatoria, donde se producirían algunas agrupaciones pero predominando la dispersión.

\begin{tabular}{|c|c|c|}
\hline $\begin{array}{l}\text { NUCLEO DE } \\
\text { POBLACION } \\
>30000 \text { hab. }\end{array}$ & $\begin{array}{l}\text { NUCLEO MÁS } \\
\text { PROXIMO }\end{array}$ & $\begin{array}{c}\text { DISTANCIA AL } \\
\text { NÚCLEO MÁS } \\
\text { PROXIMO (en } \\
\mathrm{km} \text { ) }\end{array}$ \\
\hline \multicolumn{2}{|c|}{ CASTILLA Y LEON (Núcleos $=12$} & Sup. $\left.=94224 \mathrm{~km}^{2}\right)$ \\
\hline $\begin{array}{l}\text { ARANDA DE } \\
\text { DUERO }\end{array}$ & BURGOS & 71,9 \\
\hline ÁVILA & SEGOVIA & 58,0 \\
\hline BURGOS & $\begin{array}{l}\text { MIRANDA DEL } \\
\text { EBRO }\end{array}$ & 60,2 \\
\hline LEÓN & PONFERRADA & 85,1 \\
\hline $\begin{array}{l}\text { MIRANDA DEL } \\
\text { EBRO }\end{array}$ & BURGOS & 60,2 \\
\hline PALENCIA & VALLADOLID & 42,8 \\
\hline $\begin{array}{l}\text { PONFERRAD } \\
\text { A }\end{array}$ & LEÓN & 85,1 \\
\hline SALAMANCA & ZAMORA & 60,5 \\
\hline SEGOVIA & ÁVILA & 58,0 \\
\hline SORIA & $\begin{array}{l}\text { ARANDA DE } \\
\text { DUERO }\end{array}$ & 104,6 \\
\hline VALLADOLID & PALENCIA & 42,8 \\
\hline ZAMORA & SALAMANCA & 60,5 \\
\hline \multicolumn{3}{|c|}{789,7} \\
\hline
\end{tabular}

Cuadro 10: Castilla y León( España)- Índice de Concentración - Dispersión

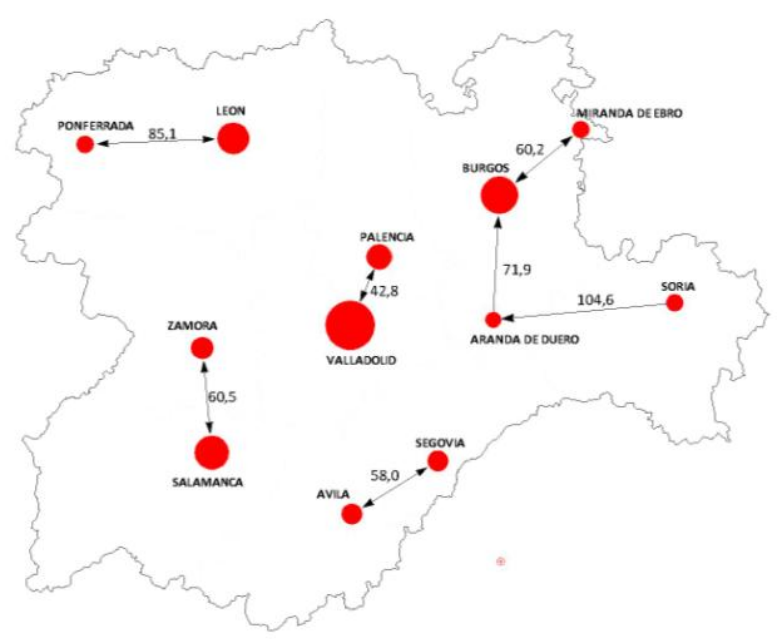

Gráfico 11: Castilla y León. Distancias al núcleo principal más próximo > 30.00o hab - Fuente: elaboración propia. Distancias tomadas en línea recta $(\mathrm{en} \mathrm{km})$.

Las flechas señalan el núcleo más próximo de cada uno de los asentamientos.

$$
\begin{aligned}
& \mathrm{d} \text { medio }=\Sigma \mathrm{d} / \mathrm{N} \\
& \mathrm{d} \text { medio CYL }=65,81 \mathrm{~km} \\
& \mathrm{Rn} \mathrm{CYL}=2 \times 65,81 \mathrm{~V} 12 / 94224=\mathbf{1 , 4 8}
\end{aligned}
$$

El valor del índice Rn puede variar entre 0 y 2,15.

En el primer caso se trataría de una distribución totalmente concentrada, en la que todos los asentamientos aparecerían en contigüidad. En el caso del índice mayor sería una distribución equilibrada. $Y$ el caso más común con un índice con valores próximos a 1 se referiría a una dispersión aleatoria, donde se producirían algunas agrupaciones pero predominando la dispersión.

Las 12 ciudades principales de Castilla y León, señaladas en el gráfico 11, presentan una distribución aleatoria con tendencia al equilibrio, debido a que las distancias entre dichos núcleos se aproximan en general al valor medio de $65,81 \mathrm{~km}$.

Las ciudades que desequilibran el sistema son Valladolid y Palencia, que se encuentran muy próximas con solo 42,8 $\mathrm{km}$. de distancia, y en otro extremo con distancias superiores, se encuentran León y Ponferrada con 85,1 km. de separación y la situación más destacable de Soria y Aranda de Duero con 104,6 $\mathrm{km}$. entre dichos núcleos. 


\section{Conclusiones}

Los índices analizados: primacía, disimilaridad, Gini, desequilibrio poblacional, desequilibrio de renta, gravitatorio, Nelson, Christaller, Davies, empleo básico, regularidad perimetral, superficial de forma y de concentración/ dispersión, constituyen indicadores que pueden permitir entender y diagnosticar cualitativa y cuantitativamente las disparidades, centralidades y marginalidades de los diferentes ámbitos territoriales.

EXISTENCIA DE TERRITORIOS DUALES


$\mathrm{Su}$ aplicación puede referirse a cualquier sistema de regiones y ciudades en diferentes partes del mundo, coadyuvando posteriormente a instrumentar políticas territoriales que disminuyan los desequilibrios de acumulación y despoblación existentes.

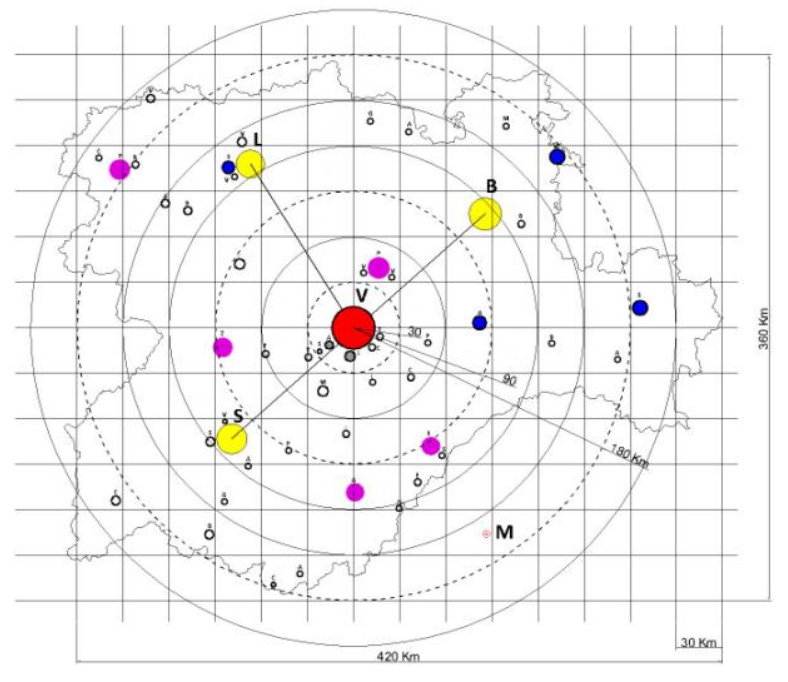

Grafico 12: Sistema de ciudades en torno a un Centro Regional

B: Burgos. S: Salamanca M: Madrid. V: Valladolid L: León

En el caso señalado en el Gráfico 12, en la constelación formada por las ciudades de la Comunidad Autónoma de Castilla y León en España, se establecen relaciones jerárquicas de dependencia, subordinación y complementariedad, con un Centro Regional (Valladolid), absorbiendo gran parte del poder político, económico y cultural del área, según la ubicación y división funcional de cada núcleo urbano, con equidistancias marcadas por el peso demográfico y económico de cada uno de ellos, medido por los diferentes indicadores definidos en el presente trabajo.(Burgos, Salamanca y León, con poblaciones en torno a $180,155, \mathrm{y}$ 133.000 habitantes respectivamente, están sensiblemente en la misma órbita, en torno a $120 \mathrm{~km}$. de la capital autónoma)

En definitiva, el diagnóstico integral de cada territorio en cualquier ámbito, local, provincial, regional $\mathrm{o}$ nacional, debería establecerse mediante el análisis de todas las variables que intervienen en sus características socioeconómicas y políticoinstitucionales, especialidad, funcionalidad, morfología, flujos de factores de producción y rol que cumple en el sistema de relaciones globales. $\mathrm{Su}$ desarrollo se mide utilizando indicadores referidos a todos los aspectos físicos (urbanísticos), (concentración, demográficos hacinamiento, etc), económicos ( ingreso y consumo per cápita), y sociales (nivel de vida, vivienda, bienestar de la población), sobre la base de estándares tipo establecidos por organismos internacionales (NNUU, UE), que cuantifican comparativamente los niveles de cobertura, calidad y satisfacción de los servicios básicos de educación, (índice de alfabetismo, o nivel de estudios), sanidad (índices de salud, mortalidad infantil, esperanza de vida), calidad y sostenibilidad medioambiental (contaminación, depuración de aguas, ruido), alimentación, vivienda, pobreza, marginalidad, desempleo, etc., marcando, a través de su valoración y evolución temporal, la caracterización político-económica de las distintas áreas como norte/sur, centro o periferia, desarrollo, infradesarrollo, etc.

Los índices tratados en este trabajo no han pretendido cubrir todo el 
amplio abanico de aspectos señalados, habiendo sido seleccionados solo los índices con una relación mas directa con la estructura física, si bien algunos de los casos estudiados, como los Índices de Gini, Desequilibrio de Rentas o de Nelsón, tienen una base económica determinante para la definición de un territorio específico. Los índices de regularidad perimetral y superficial de forma, quieren incidir en las ventajas sociales y económicas de la concentración urbana en los nuevas ordenaciones urbanísticas, frente a las tendencias dispersadoras y fragmentadoras de la ciudad contemporánea.

\section{Referencias}

ACEBILLO José A y otros (2002): Redes, territorios y gobierno: Nuevas respuestas globales a los retos de la globalización. Edit. Diputación de Barcelona 2002

AROCHE Fidel (2003): Notas para una reflexión sobre desarrollo regional. Actualidad Económica-Perú.

AMIN Ash y Thrift Nigel (2002): Redes, Territorios y gobierno: Repensando la teoría urbana. Edit. Diput. Barcelona

BORJA-CASTELLS (2004): Local y Global, la gestión de las ciudades en la era de la información: Edit. Santillana Edit. Generales Madrid

BREESE Gerald, SURINDER K. Mahta(1974): La ciudad en los países en vías de desarrollo, Edit. Tecnos Madrid

CAMAGNI Roberto: Economía Urbana.( 2004) Edit. R. Boix

CAPEL, Horacio (1968): Estructura funcional de las ciudades españolas en 1950. Revista de Geografía

DAVIES, Wayne. Centrality and the central place hierarchy. Urban Studies. Enero 1967. Vol. 4 (1). págs. 61-79.
GUTIÉRREZ PUEBLA, Javier (1984): "La Ciudad y la Organización Regional", Cuadernos de estudio, Serie Geografía No ${ }^{0}$. 14. Edit. CINCEL. Madrid,

HAGGET(1976): Análisis Locacional en Geografía Humana. Gustavo Gili. Barcelona

RACIONERO Luis (1981): Sistemas de ciudades y ordenación del territorio. Alianza . Madrid

SANZ CAÑADA E (1981): La ordenación del territorio y el sistema de ciudades. Un caso de aplicación de técnicas multivariantes a la definición del sistema urbano. Estudios territoriales $\mathrm{n}^{0} 1$ pag. $63-69$

VEGARA GÓMEZ, Alfonso y Introducción al análisis demográfico : un enfoque para urbanistas. Taller de Ideas, Centro de Estudios Urbanos, D.L. Pamplona, pp. 106-107.

\section{Notas}

\begin{abstract}
GUTIÉRREZ PUEBLA, Javier: "La Ciudad y la Organización Regional", Cuadernos de estudio, Serie Geografía №. 14. Edit. CINCEL. Madrid, 1984. En el libro se cita el caso de Uruguay, relacionando las ciudades de Montevideo, salto, Paysandú y las Piedras, con un indice de primacía (año 1975) de 86,80 reflejando la macrocefalia de Montevideo, y el caso de Perú, Ip: 79,7., con Índices característicos de países que formaban parte de un imperio colonial, con sistemas de ciudades poco articulados.
\end{abstract}

\footnotetext{
ii Fuente demográfica: INE 1/1/2011

iii ZÁRATE MARTíN y RUBIO BENITO, 2005. www.uclm.es/.../prácticas\%20Geografia\%20UE\%20ciud ades\%2007

iv VEGARA GÓMEZ, Alfonso y D’ENTREMONT, Alban: Introducción al análisis demográfico : un enfoque para urbanistas. Taller de Ideas, Centro de Estudios Urbanos, D.L. Pamplona, pp. 106-1071988.
}

\footnotetext{
${ }^{\vee}$ Duncan, O. D. \& B. Duncan (1955). "A methodological analysis of segregation indices", American Sociological Review

vi Ver aplicación al caso Chileno en el trabajo "Las migraciones intraurbanas y su impacto en la
} 
diferenciación del espacio social del Gran Santiago" de Ortiz, J. \& P. Schiappacasse (1997). Revista Geográfica de Chile Terra Australis, 42. Y referido a Barcelona, el trabajo de Carles Martori i Cañas Joan y Hoberg Karen: Indicadores Cuantitativos de Segregación Residencial. El caso de la población inmigrante en Barcelona. Geo Crítica / Scripta Nova. Revista electrónica de geografía y ciencias sociales. Barcelona: Universidad de Barcelona, 15 de julio de 2004, vol. VIII, núm. 169. <http://www.ub.es/geocrit/sn/sn-169.htm>

vii GINI Corrado (1884- 1965) Estadístico, demógrafo y sociólogo italiano desarrolló el coeficiente que lleva su nombre como una medida de la desigualdad en los ingresos en una sociedad. En este caso lo aplicamos a la relación entre población y territorio.

viii Gráfico 6. Indice de Convergencia respecto a UE15. Año 2008. Balance Económico Regional 2000-2008; FUNCAS, enero 2009

${ }^{i x}$ GINI Corrado (1884-1965) Estadístico, demógrafo y sociólogo italiano desarrolló el coeficiente que lleva su nombre como una medida de la desigualdad en los ingresos en una sociedad. En este caso lo aplicamos a la relación entre población y territorio.

× GUTIERREZ PUEBLA, Javier (1992). Obra citada

${ }^{x i}$ EMPLEO NORMAL: proporción de empleo en cada una de las ramas de actividad económica que se consideren normales en las distintas ciudades del sistema de estudio y por ramas de actividad.

xii Para la evaluar el grado de concentración/dispersión en el caso del hábitat rural se emplean además otros índices como el Índice de Colas que mide el grado de concentración de la población en un territorio dado y permite compararla con la de los distintos municipios de un área y se puede expresar mediante el cociente: $\mathrm{K}=[\mathrm{S}$ $x \quad N] / \quad[T-P d]$, representando $K$ el nivel de concentración, Pd la población total dispersa (sin contar la población del mayor lugar del área), $\mathrm{N}$ el número de lugares poblados menos $1, \mathrm{~T}$ la población total y $\mathrm{S}$ la superficie. Cuanto más bajo sea el valor del índice tanto mayor será el grado de concentración. El numerador expresa la tendencia a la dispersión, el denominador expresa la tendencia a la concentración. Los resultados expresarán: 0 a 0,1: Concentración máxima, 0,1 a 0,2 Concentración media, Más de 0,2: Concentración mínima.

El Índice de Demageon relaciona la población total de los lugares poblados y el número de lugares, con la población de la unidad territorial estudiada. $\mathrm{K}=$ $\mathrm{P} \mathrm{dx} \quad \mathrm{N} / \mathrm{T}$, donde $\mathrm{Pd}$ es la población total dispersa (sin contar la del mayor lugar del área), $\mathrm{N}$ el número de lugares poblados menos 1 y T la población total.

El Índice de Bernard introduce además el factor viviendas, complementando los índices de Colas y Demageon. La fórmula de este índice es: $\mathrm{K}=\mathrm{C} \times \mathrm{S} / \mathrm{N} 2$. donde $\mathrm{C}$ es el numero de viviendas existentes en la circunscripción; S: la superficie en km2 y $\mathrm{N}$ el número de lugares poblados. 